\title{
Динамические характеристики микротермохимического детектора для газовой хроматографии
}

\author{
Платонов И.А., Платонов Вл.И., Платонов Вал.И., Горюнов М.Г. \\ Самарский национальный исследовательский университет имени академика С.П. Королева, Самара \\ Поступила в редакцию 13.11.2017 г.
}

DOI: https://doi.org/10.17308/sorpchrom.2018.18/531

Разработан микротермохимический детектор для газовой хроматографии, обеспечивающий работу с капиллярными, микрокапиллрными и поликапиллярными МЭМС колонами. Проведено сравнение основных технических и динамических характеристик разработанного детектора и с традиционно используемыми в практике газохроматографического анализа детекторами по ионизации в пламени, проволочным детектором по теплопроводности и пленочным микродетектром по теплопроводности. Установлено, что презиционность анализа с использованием предлагаемого микротермохимического детектора увеличивается в среднем на $30 \%$, предел детектирования снижается в 100 раз, при этом значительно улучшается инерционность детектора, что обеспечивает возможность его использования при проведении высокоскоростных измерений в составе портативных аналитических приборов на основе микрофлюидных систем.

Ключевые слова: газовая хроматография, микротермохимический детектор, микроэлектромеханические системы, предел детектирования, уровень флуктуационных шумов, инерционность.

\section{Dynamic characteristics of a microthermochemical detector for gas chromatography}

\author{
Platonov I.A., Platonov V1.I., Platonov Val.I., Goryunov M.G. \\ Samara National Research University, Samara
}

Since its introduction in 1952, the method of gas chromatography has found wide application in various laboratories whose needs led to the development of a variety of detection devices. To date, about 50 detectors with universal, selective and specific characteristics have been proposed for gas chromatography.

Pronounced tendency of modern analytical chemistry, including gas chromatography, is miniaturization chromatography apparatus (columns, dispensing devices, detectors) allowing operatively perform complex analyzes of natural and manmade objects on MEMS low capacitance column using detectors, fabricated using silicon technology [1]. One of these detectors is a microcircuit based on film thermo-sensitive elements, the main technical characteristics of which were considered in [2-3].

For the purpose of increase in sensitivity and a precision of detecting the micro-thermochemical detector has been developed for a gas chromatography. The detector has working and comparative sensitive elements which are included in the bridge measuring scheme and are in one working camera of the detector, no more than $0.15 \mathrm{ml}$. Besides, the detector has two directing screens located parallel to platinum threads and also the calibrated sections for uniform distribution of a gas stream to working and comparative platinum threads. The developed detector allows to reduce considerably the level of fluctuation noise and drift of a zero signal due to compensation of influence of change of temperature, a consumption of gas carrier, supply voltage, etc. 
Use of the differential bridge scheme when working and comparative platinum threads are in one flowing camera of the detector, allows to raise a limit of detecting of the microthermochemical detector on two orders in comparison with the known thermochemical detector of the gas chromatograph the Crystal of 5000.1 (Hromatek). Results of pilot studies have shown that use of MTCHD allows to increase for $10 \%$ lag effect in comparison with MTCD and to approach almost inertialess detector on ionization in a flame

Keywords: gas chromatography, microthermochemical detector, microelectromechanical systems, detection limit, level of fluctuation noise, inertia.

\section{Введение}

С момента своего появления в 1952 г. метод газовой хроматографии нашел широкое применение в различных лабораториях, потребности которых привели к разработке разнообразных вариантов детектирующих устройств. На сегодняшний день для газовой хроматографии предложено около 50 детекторов с универсальными, селективными и специфическими характеристиками.

Ярко выраженной тенденцией современной аналитической химии, в том числе газовой хроматографии, является миниатюризацию хроматографической аппаратуры (колонок, дозирующих устройств, детекторов), позволяющей оперативно проводить сложные анализы природных и техногенных объектов на МЭМС колонках малой емкости с использованием детекторов, изготовленных с использованием кремниевых технологий [1]. Одним из таких детекторов является микрокатарометр на основе пленочных термочувствительных элементов, основные технические характеристики которого были рассмотрены в работах [2-3].

Несмотря на то, что разработанный авторами детектор по быстродействию в несколько раз превосходит проволочные ДТП и имеет предел детектирования в два раза ниже предела детектирования штатного детектора по теплопроводности таких хроматографов, как «Кристалл 2000» и «Кристалл 5000». Тем не менее, этот детектор имеет инерционность, значительно уступающую инерционности детектора по ионизации в пламени, а, следовательно, не может работать в режиме высокоскоростной газовой хроматографии [4] (когда ширина хроматографического пика должна составлять 30-200 мсек.). В этой связи наиболее интересным вариантом является изучение возможности использования в портативных газовых хроматографах микротермохимического детектора в сочетании с МЭМС колонками различной геометрии, которые обеспечивают высокоскоростной анализ с целью определения содержания горючих газов и кислорода в сложных смесях веществ природного и техногенного происхождения [5].

Термохимические детекторы были использованы в серийных газовых хроматографах типа ХТ и ХТП начиная с 1962 г. Данный детектор состоял из металлического корпуса с рабочей и сравнительной камерами, в которых были установлены термокаталитические чувствительные элементы, выполненные в виде платиновых спиралей включенных в мостовую схему измерения. Однако такие термохимические детекторы обладают относительно низкой надежностью и стабильностью из-за высокой температуры чувствительных элементов, которая достигает температуры более $900^{\circ} \mathrm{C}$ при рабочих условиях в результате того, что рабочий чувствительный элемент одновременно выполняет функции катализатора реакций окисления горючих компонентов и измерителя температуры в мостовой измерительной схеме [6]. В настоящее время, в практике газоаналитических измерений применяют комбинированные чувствительные элементы, в которых разделены функции термокаталитической реакции и измерения температуры. Этот эффект обеспечивается за счет того, что на платиновую спираль или нить наносят эффективное каталитическое платиново-палладиевое покрытие с разветвленной поверхностью, на которой происходит окисление горючих компонентов при значительно меньших температурах $400-500^{\circ} \mathrm{C}$,

Платонов и др. / Сорбционные и хроматографические процессы. 2018. Т. 18. № 3 
чем на чистой платине [7]. Впервые чувствительные элементы изготовленные по новой технологии были использованы в низкотемпературном детекторе каталитического горения для газового хроматографа XТ-4 для анализа продуктов сгорания топливоиспользующих устройств [8]. Недостатком низкотемпературного детектора каталитического горения является повышенная инерционность, которая исключала его использование при детектировании сигнала в газохроматографических системах с капиллярными и микронасадочными колонками. Большая инерционность вызвана с одной стороны большой величиной мертвого объема камеры детектора, а с другой стороны длительностью процесса установления теплового равновесия в газовом потоке с нагретыми термочувствительными элементами.

\section{Эксперимент}

С целью повышения чувствительности и прецизионности детектирования нами был разработан микротермохимческий детектор для газовой хроматографии, содержащий рабочий и сравнительный чувствительные элементы в виде платиновых нитей, одна из которых покрыта тонким слоем катализатора, которые включены в мостовую измерительную схему. В качестве рабочего и сравнительного чувствительных элементов используется термохимический датчик ДТЭ-1, в цилиндрическом колпачке которого высверлены два диаметрально расположенных отверстия. Одно из отверстий служит для капилляра, подводящего элюат из разделительной колонки, который снабжен двумя направляющими экранами, расположенными параллельно платиновым нитям и имеет сечение для равномерного распределения газового потока из колонки на рабочую и сравнительную платиновую нити. Второй отверстие служит для выхода элюата из детектора. На рисунке 1 представлена принципиальная схема предлагаемой конструкции детектора.

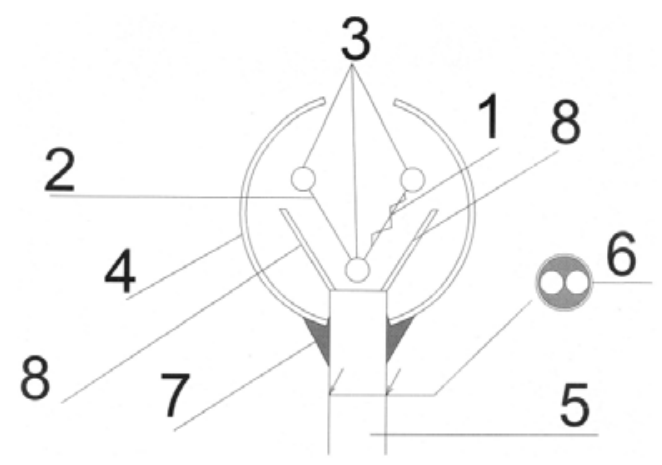

Рис. 1. Принципиальная схема микротермохимического детектора:

1 - рабочий чувствительный элемент; 2 - сравнительный чувствительный элемент; 3 - выходное отверстие; 4 - рабочая камера детектора; 5 - подводящий капилляр; 6 - сечение капилляра; 7 - герметизация канала; 8 - направляющий экран.

Использование такой конструкции позволяет значительно уменьшить уровень флуктуационных шумов и дрейф нулевого сигнала за счет компенсации влияния изменения различных параметров (температура, расхода газа-носителя, напряжения питания и др.) при дифференциальной мостовой схеме измерения, когда рабочая и сравнительная платиновые нити расположены в одной проточной камере детектора. Повышение чувствительности обеспечивается также за счет того, что подводящий элюат капилляр имеет направляющие экраны, а также сечения для равномерного распределения газового потока из колонки на рабочую и сравнительную платиновую нити.

Платонов и др. / Сорбционные и хроматографические процессы. 2018. Т. 18. № 3 
Экспериментальная оценка чувствительности и прецизионности детектирования известного и предлагаемого термохимических детекторов проводилась на примере анализа поверочных газовых смесей (ПГС-2 разряда) «водород в воздухе» и «пропан в воздухе» с концентрацией $0.2 \%$ об. на газовом хроматографе «Кристалл $5000.1 »$ (ЗАО СКБ «Хроматэк») с капиллярной колонкой из алюминия на плоскости, заполненная нанодисперсным диоксидом кремния (Аэросил Ф-175), длина 2 м, сечение -0.2 мм $\times 0.2$ мм. Температура колонки, $\mathrm{T}_{\mathrm{c}}=40^{\circ} \mathrm{C}$, газ-носитель - воздух, расход газа-носителя в колонке $\mathrm{F}_{\mathrm{c}}=2 \mathrm{~cm}^{3} /$ мин при делении потока 1:50. Температура детектора $50^{\circ} \mathrm{C}$.

По результатам анализа были рассчитаны:

1. Уровень флуктуационных шумов, $\Delta x^{\prime}$, В. Для этого производилась запись сигнала без ввода пробы в течение 10 мин.

$$
\Delta x^{\prime}=\frac{\Delta x}{K_{\Pi P}},
$$

где $\Delta x^{\prime}$ - максимальное значение амплитуды повторяющихся колебаний нулевого сигнала, с полупериодом (длительностью импульса), не превышающим 10 с., при этом колебания, имеющие характер одиночных импульсов длительностью не более 1 с. не учитывались; К нала, равный $3.2 \cdot 10^{6} \mathrm{~B}^{-1}$.

2. Дрейф нулевого сигнала, $\Delta y^{\prime}$ В/ч. Без ввода пробы записывается хроматограмма нулевого сигнала. За дрейф принимается наибольшее смещение уровня нулевого сигнала в течение 1 часа.

$$
\Delta y^{\prime}=\frac{\Delta y}{K_{\Pi P}},
$$

где $\Delta y$ - смещение уровня нулевого сигнала, на выходе усилителя, В.

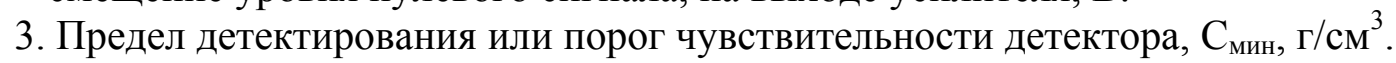

$$
C_{\text {мин }}=\frac{2 \Delta x^{\prime}}{\bar{A} \cdot F_{c}}
$$

где $\mathrm{m}$ - масса водорода в дозе хроматографа, г; $\bar{A}$ - среднее арифметическое значение площади пика водорода, $\mathrm{B} \cdot \mathrm{c} ; \mathrm{F}_{\mathrm{C}}$ - расход газа-носителя на выходе колонки, $\mathrm{cm}^{3} / \mathrm{c}$.

4. Относительное среднеквадратичное отклонение (СКО), \% для площади пи-

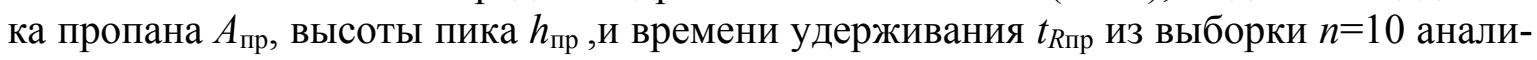
зов ПГС пропана в воздухе.

$$
S_{g}=\frac{1}{g_{\Pi Р}} \cdot 100 \sqrt{\frac{\sum_{1}^{n}\left(g_{\Pi Р}-\overline{g_{\Pi P}}\right)^{2}}{n-1}},
$$

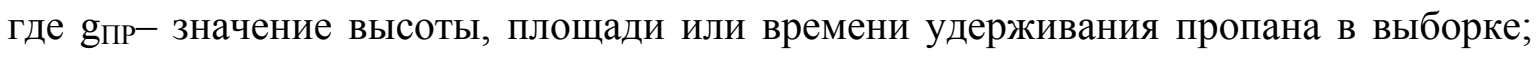
$\overline{g_{\text {ПР }}}$ - среднее арифметическое значение измеряемых величин.

Сравнительная оценка по инерционности разработанного микротермохимического детектора (МТХД) проводилась по отношению к пленочному микродетектору по теплопроводности (МДТП) и практически безынерционному детектору по ионизации в пламени (ДИП) на газовом хроматографе «Hewlett-Packard» модели 5890. С использованием одного из методов, предложенного в работе [2], который заключается в оценке инерционности по величине степени разделения $\mathrm{R}_{\mathrm{s}}$ для хроматографических пиков изо-бутана и нормального бутана по уравнению (5):

$$
R_{S}=\frac{\Delta l}{w_{h 1}+w_{h 2}},
$$


Эксперимент проводили с использованием планарной МЭМС колонки сечением 0.6×0.6 мм, длиной 1 м, заполненной адсорбентом Carbopack B с зернением 100-120 МЕШ и газовой смесью углеводородов (пропан-бутановая фракция). Температура термостата колонок $60^{\circ} \mathrm{C}$, температура термостата пленочного детектора по теплопроводности и микротермохимического детектора составляла $27^{\circ} \mathrm{C}$, температура термостата детектора по ионизации в пламени составляла $150^{\circ} \mathrm{C}$. Расход газаносителя (воздух) во всех случаях составлял $5 \mathrm{~cm}^{3} /$ мин, объем вводимой пробы - 50 мкл.

\section{Обсуждение результатов}

Результаты эксперимента по определению динамических характеристик детекторов представлены в табл. 1.

Таблица 1. Сравнительные данные экспериментальной проверки известного и предлагаемого детекторов

\begin{tabular}{|c|c|c|c|c|c|c|}
\hline \multirow{2}{*}{ Наименование детектора } & \multicolumn{3}{|c|}{$\begin{array}{c}\text { Поверочная газовая смесь } \\
\text { «водород в воздухе» }\end{array}$} & \multicolumn{3}{|c|}{$\begin{array}{c}\text { Поверочная газовая смесь } \\
\text { «пропан в воздухе» }\end{array}$} \\
\hline & $\begin{array}{c}\Delta x^{\prime} \\
\mathrm{B}\end{array}$ & $\begin{array}{l}\Delta y^{\prime} \\
\mathrm{B} / \mathrm{ч}\end{array}$ & $\begin{array}{l}\mathrm{C}_{\text {мин }} \\
\Gamma / \mathrm{cm}^{3}\end{array}$ & $\begin{array}{c}\mathrm{S}_{\text {Апр }}, \\
\%\end{array}$ & $\begin{array}{l}\mathrm{S}_{\mathrm{hnp}}, \\
\%\end{array}$ & $\begin{array}{c}\mathrm{S}_{\mathrm{tR} \text { Rp }} \\
\%\end{array}$ \\
\hline $\begin{array}{c}\text { Известный термохимиче- } \\
\text { ский детектор ДТХ ЗАО } \\
\text { СКБ «Хроматэк» }\end{array}$ & $6.0 \cdot 10^{-6}$ & $1.2 \cdot 10^{-4}$ & $3.0 \cdot 10^{-10}$ & 2.0 & 2.0 & 2.0 \\
\hline $\begin{array}{c}\text { Предлагаемый микротермо- } \\
\text { химический детектор для } \\
\text { газовой хроматографии }\end{array}$ & $9.4 \cdot 10^{-8}$ & $5.0 \cdot 10^{-6}$ & $3.0 \cdot 10^{-12}$ & 0.4 & 0.5 & 0.5 \\
\hline
\end{tabular}

Как видно из приведенных в таблице 2 данных, предлагаемый термохимический детектор для газовой хроматографии имеет уровень флуктуационных шумов почти на два порядка меньше, чем известный детектор ДТХ серийно выпускаемого газового хроматографа «Кристалл-5000», благодаря чему удалось увеличить на два порядка чувствительность предлагаемого детектора. Прецизионность предлагаемого детектора улучшилась в среднем в 1.3 раз. На рис. 2 представлена хроматограмма разделения пропана, изо-бутана и н-бутана с использованием трех различных детекторов.

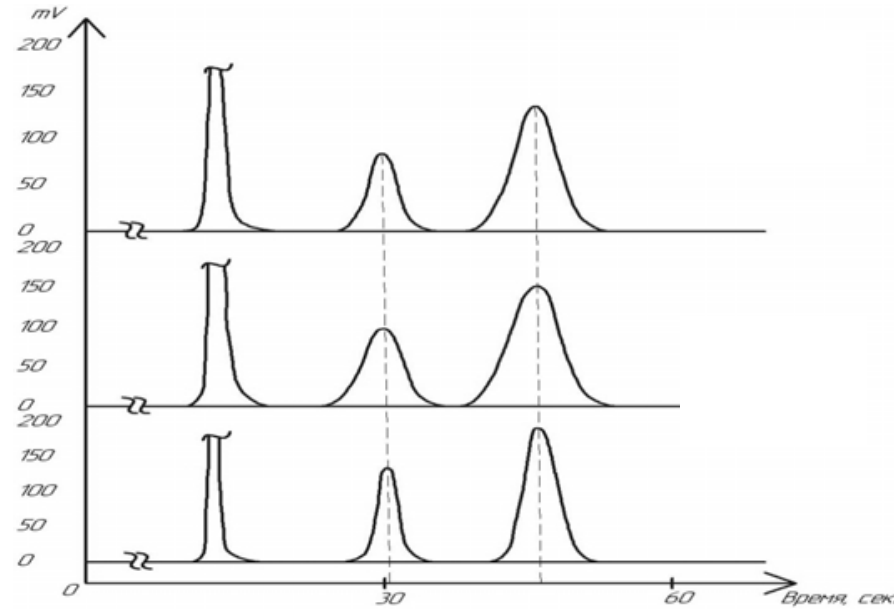

Рис. 2. Хроматограммы разделения пропана, изо-бутана и н-бутана: а - МДТП, б - МТХД, в - ДИП 

в табл. 2.

Результаты эксперимента по оценке инерционности детекторов представлены

Таблица 2. Результаты оценки инерционности

\begin{tabular}{|c|c|c|c|c|}
\hline Детектор & $\Delta l, \mathrm{c}$ & $\mathrm{w}_{\mathrm{h} 1}$ & $\mathrm{w}_{\mathrm{h} 2}$ & $\mathrm{R}_{\mathrm{S}}$ \\
\hline \multirow{4}{*}{ мдТП } & 19.10 & 4.10 & 8.30 & 1.54 \\
\hline & 19.90 & 4.10 & 8.20 & 1.62 \\
\hline & 20.10 & 4.20 & 7.60 & 1.70 \\
\hline & \multicolumn{3}{|c|}{ Среднее } & 1.62 \\
\hline \multirow{4}{*}{ МТХД } & 17.30 & 3.90 & 6.00 & 1.75 \\
\hline & 17.20 & 3.80 & 5.90 & 1.77 \\
\hline & 17.20 & 3.80 & 6.00 & 1.76 \\
\hline & \multicolumn{3}{|c|}{ Среднее } & 1.76 \\
\hline \multirow{4}{*}{ ДИП } & 23.40 & 4.92 & 7.62 & 1.87 \\
\hline & 24.00 & 4.92 & 7.62 & 1.91 \\
\hline & 24.00 & 4.86 & 7.62 & 1.92 \\
\hline & \multicolumn{3}{|c|}{ Среднее } & 1.90 \\
\hline
\end{tabular}

Как видно из таблицы 2 , использование разработанного МТХД для газовой хроматографии позволяет повысить инерционность по сравнению с МДТП на $11 \%$ и приблизится по инерционности к практически безынерционному ДИП. Использование предлагаемого термохимического детектора для газовой хроматографии позволяет:

1. Значительно повысить чувствительность и прецизионность количественных измерений при работе с капиллярными и микронасадочными колонками.

2. Заменить пламенно-ионизационный детектор более простым термохимическим детектором в газовых микрохроматографах.

\section{Заключение}

В рамках работы установлено, что презиционность анализа с использованием предлагаемого микротермохимического детектора увеличивается в среднем на $30 \%$, предел детектирования снижается в 100 раз, при этом значительно улучшается инерционность детектора, что обеспечивает возможность его использования при проведении высокоскоростных измерений в составе портативных аналитических приборов на основе микрофлюидных систем.

Работа выполнена при финансовой поддержке

Российского фонда фундаментальных исследований (проект № 17-43-630873).

\section{Список литературы}

1. Золотов Ю.А. // Успехи химии. 2006. № 75 (4). С. 299-301.

2. Платонов И.А., Арутюнов Ю.И. // Becmник СамГУ. 2006. № 6/1. С. 280-291.

3. Платонов И.А., Ланге П.К., Колесниченко И.Н., Платонов В.И. // Измерительная техника. 2015. № 5. С. 72-74.

4. Van Deursen M.M., Beens J., Janseen H.-G. et al. // J. Chromatog. 2000. Vol. 878. pp. 205208.
5. Sidelnikov V.N., Nikolaeva O.A, Platonov I.A., Parmon V.N. // Russ. Chem. Rev. 2016. № 85 (10). pp.1033-1055.

6. Жуховицкий А.Н., Туркельтауб Н.М. Газовая хроматография. М. Гостоптехиздат. 1962. $442 \mathrm{c}$.

7. Карпов А.В., Карпов Е.Ф., Кавченко В.С., Михайлов В.Н. и др. Способ изготовления чувствительных элементов. Авт. Свид. СССР № 293499 от 03.11.1970 г. по заявке 
института горного дела им. Скочинского №1318109 от 10.01.1969 г.

\section{References}

1. Zolotov YU.A., Uspekhi khimii, 2006, No 75 (4), pp. 299-301.

2. Platonov I.A., Arutyunov YU.I., Vestnik SamGU, 2006, No 6/1, pp. 280-291.

3. Platonov I.A., Lange P.K., Kolesnichenko I.N., Platonov V.I., Izmeritel'naya tekhnika, 2015, No 5, pp. 72-74.

4. Van Deursen M.M., Beens J., Janseen H.-G. et al., J. Chromatog., 2000, Vol. 878, pp. 205208.

5. Sidelnikov V.N., Nikolaeva O.A, Platonov I.A., Parmon V.N.,/ Russ. Chem. Rev., 2016, No 85 (10), pp. 1033-1055.

Платонов Игорь Артемьевич - д.т.н., профессор, самарский университет, Самара

Платонов Владимир Игоревич - к.х.н., доцент, самарский университет, Самара

Платонов Валерий Игоревич - аспирант, самарский университет, Самара
8. Арутюнов Ю.И. Хроматографическое измерение состава нефтяных газов. М. Недра. 1987. С. 196-198.

6. ZHukhovitskiy A.N., Turkel'taub N.M., Gazovaya khromatografiya, M., Gostoptekhizdat, 1962, $442 \mathrm{p}$.

7. Karpov A.V., Karpov E.F., Kavchenko V.S., Mikhaylov V.N. et al., Sposob izgotovleniya chuvstvitel'nykh ehlementov, Avt. Svid. SSSR № 293499 ot 03.11.1970 g. po zayavke instituta gornogo dela im. Skochinskogo №1318109 ot $10.01 .1969 \mathrm{~g}$.

8. Arutyunov YU.I., KHromatograficheskoe izmerenie sostava neftyanykh gazov, M., Nedra, 1987, pp. 196-198

Platonov Igor A. - doctor of technical Sciences, Professor, Samara University, Samara

Platonov Vladimir I. - Ph.D., Associate Professor, Samara University, Samara

Platonov Valery I. - post-graduate student, Samara University, Samara 